\title{
Role of mood and connotation in the spacing effect
}

\author{
DAVID G. ELMES, PAUL F. CHAPMAN, and CAMDEN W. SELIG \\ Washington and Lee University, Lexington, Virginia
}

\begin{abstract}
Half of the 36 subjects in this study underwent a procedure designed to induce a depressed mood, and a neutral mood was induced in the other half of the subjects. All subjects then heard and attempted to free-recall a word list that contained words of neutral, good, and bad connotation. The words were presented once within the list, or they were presented twice with spacing intervals of 0 or 7 . Neither mood nor connotation had a main effect on the number of words recalled. Depressed subjects showed a spacing effect for the affectively loaded words but not for the neutral ones. In contrast, the nondepressed subjects showed a spacing effect for the neutral words but not for the affectively toned ones.
\end{abstract}

Over the past dozen years or so, a substantial amount of research has tried to determine why spaced repetitions lead to better retention than do massed repetitions (for a review, see Glenberg, 1979). The superior recall of spaced items is called the spacing effect, and the main focus of research on it has concerned acquisition, storage, and retrieval-what the social psychologists (e.g., Zajonc, 1980) call "cold" cognitive processes. A recent series of studies from our laboratory yielded results that implicate "hot" cognitive processes in the spacing effect (Elmes, Dye, \& Herdelin, 1983). We have found that subjects simply do not like nominally neutral material that has been studied under massed practice as much as they do the same material that has been studied either once or under spaced practice.

We are not sure how this negative affect for material studied under massed practice enters into retention. One possibility revolves around the mood-congruence effect demonstrated by Bower (1981). The moodcongruence effect is a state-dependent memory based on mood in which sad events are remembered best when a person is sad and happy events are remembered best when a person is happy. With regard to the spacing effect, it could be that since the typical subject in the typical memory experiment is in a neutral mood as he or she is memorizing massed and spaced items, the unpleasant massed items are recalled poorly because they do not match the mood of the memorizer. From this it follows that a memorizer who is unhappy or depressed may show a smaller difference in the levels of massed and spaced words that are recalled than is usually observed-the spacing effect should be attenuated when the subject is depressed. We tested this possibility by

Portions of these data were reported at the meeting of the Psychonomic Society, San Diego, November 1983. This research was supported by Robert E. Lee research grants. Direct correspondence to David G. Elmes, Department of Psychology, Washington and Lee University, Lexington, VA 24450. making some subjects depressed and determining their level of recall for massed and spaced words. In addition, the to-be-remembered words had a neutral, good, or bad connotation, according to the semantic differential (Jenkins, 1960). The expectation was that the effects of connotation would depend on the mood of the memorizer, because earlier results (Elmes et al., 1983) had indicated no spacing effect for good and bad words when the participant's mood had not been altered (i.e., was neutral).

\section{METHOD}

\section{Subjects}

The subjects were 36 male undergraduates at Washington and Lee University. They volunteered to participate in order to receive extra credit in introductory psychology courses. When they agreed to participate, they were cautioned that some aspects of the experimental procedure might be disturbing, though not physically painful, and that they could decline to participate if they wished.

\section{Design}

Mood was varied between subjects. Half of the subjects were assigned randomly to the "depressed" group, in which they underwent a procedure designed to induce a depressed mood. The remaining half of the subjects engaged in a task that was designed to maintain a neutral mood-the "neutral" group. Presentation condition and connotative meaning of the to-beremembered items were varied within subjects.

\section{Materials}

List construction. The list structure was identical to that used by Elmes et al. (1983). The 18 target words were presented once, or they were repeated at spacing intervals of 0 or 7 words. Twelve filler words served as buffers for primacy and recency effects and as within-list fillers to maintain appropriate spacing. Different list forms were devised so that each presentation cơndition immediately preceded the first recency-buffer word. The target items were one- and two-syllable nouns selected from the connotative meaning norms provided by Jenkins (1960). The 6 "good" words had a mean rating of 2.13 , the 6 "neutral" words had a mean rating of 3.45 , and the 6 "bad" words had a mean rating of 5.21. None of the connotative meaning ratings overlapped between these three groups. Across subjects, conno- 
tative meaning was completely counterbalanced among the prerecency list forms.

Mood assessment. The subjects were initially screened by means of the Beck Depression Inventory (Beck, 1967). Anyone who scored higher than 15 was excused from participation and given full extra credit. Self-rating scales required the subjects to make a vertical mark on a $100-\mathrm{mm}$-long horizontal line to indicate their mood "at this moment." One scale was bounded by "I do not feel at all anxious" on the left and by "I feel extremely anxious" on the right. Two other scales replaced the word "anxious" with either "happy" or "despondent." Scoring involved finding the distance of the vertical mark from the left end of the scale (to the nearest millimeter). The subjects also engaged in a writing-speed test in which they were required to write backward from the number 100 by ones for $1 \mathrm{~min}$.

Mood induction. Velten's (1968) mood-induction task involves reading 60 statements, first silently and then aloud. The 18 students in the "depressed" group read self-referent statements designed to induce a depressed mood. The sentences became progressively more depressing as the subject proceeded through the series. One of the more depressing statements was "All of the unhappiness of my past life is taking hold of me." The subjects in the "neutral" group read innocuous statements that were not self-referent (e.g., "The campus covers some 600 acres").

\section{Procedure}

Initially, the subjects were told about the general nature of the experiment and were told that they could discontinue participation at any time they wished and still receive full extra credit. After a baseline writing-speed measure, the subjects completed the Beck Depression Inventory. Then the subjects answered a few questions about their experience with psychotherapy. Subjects who indicated that they were currently undergoing therapy were excused from participation. At this time, they also completed the magnitude estimation scales for their current mood along the dimensions of happiness, anxiety, and despondency. Following the mood-induction procedure, the subjects heard the list of words and then attempted free recall. The words were presented via a tape recorder. The words were read by a male at a $3-\mathrm{sec}$ rate. Unlimited time was allowed for written free recall.

Following recall, validity checks for the mood induction were undertaken. These checks first involved a readministration of the writing-speed test and then a reestimate of mood along the dimensions of anxiety, happiness, and despondency.

After a thorough debriefing, the subjects in the "depressed" group read through a series of self-referent statements designed to elevate their mood (Velten, 1968). Then they signed a statement indicating that they felt no worse at the end of the experiment than they had at the beginning. The subjects in the "depressed" group were also given a list of emergency telephone numbers in case they felt depressed following the experimental session. The next day, one of the experimenters telephoned the participants in the "depressed" group to ensure that they were not depressed.

\section{RESULTS AND DISCUSSION}

To determine whether the Velten (1968) procedure influenced mood, difference scores of writing speed and mood ratings before and after mood induction were calculated. The change in anxiety following mood induction was about the same for the two groups [t(34) = .09]. The rated happiness in the "neutral" group increased across the two tests (mean $=+5.2 \mathrm{~mm}$ ), and the rated happiness in the "depressed" group decreased $($ mean $=-10.9 \mathrm{~mm})$, a significant effect $[\mathrm{t}(34)=2.79$, $\left.\mathrm{p}<.01, \mathrm{r}_{\mathrm{pb}}=.41\right]$. Estimates along the despondency dimension increased more for the subjects in the "depressed" group than for the subjects in the "neutral" group, but this difference was small and not reliable $\left[\mathrm{t}(34)=1.36, \mathrm{p}=.18, \mathrm{r}_{\mathrm{pb}}=.17\right]$. The small effect here probably occurred because many subjects said they were unsure of the meaning of "despondent." Several subjects who did not ask about the meaning of "despondent" had happiness and despondency ratings that covaried directly. Writing speed increased across tests in both groups. Although the mean speed increased more in the "neutral" group than in the "depressed" group (1.8 and 1.3 digits, respectively), this difference was not reliable $[\mathrm{t}(34)=.32]$. In summary, the Velten procedure seems to have induced a mild depression in mood: Self-ratings of happiness decreased, and there was a modest relative decline in writing speed.

As far as free recall is concerned, neither mood induction nor connotative meaning had a main effect on recall (Fs $<1)$. Leight and Ellis $(1981)$ reported that a depressed mood lowered the level of recall, but such an effect was not seen in the present experiment. Elmes et al. (1983) also reported that connotative meaning had minor effects on the level of recall. Unlike the present experiment, the one reported by Elmes et al. used a between-subjects design. Presentation condition had a marked overall effect on recall: Items repeated at a spacing of seven words were recalled better than were massed words, which in turn were recalled better than were words presented once $[F(2,68)=11.28, p=.0002$, $\eta=.49]$.

Shown in Figure 1 are the recall data as a function of presentation condition. Because the recall of good and bad words was essentially the same across conditions, their effects are combined in the figure and are illustrated under the heading "Affective Words." In the lefthand panel, there is a spacing effect for neutral words for subjects in the "neutral" group, but subjects in the "depressed" group were not responsive to spacing of the

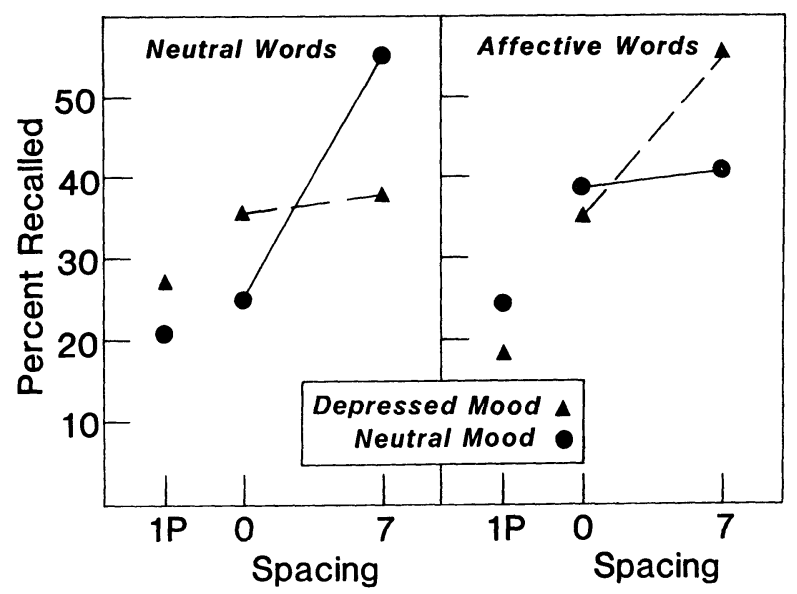

Figure 1. Mean percentage recalled as a function of presentation condition, word connotation, and mood. 
neutral words. Thus, depressing subjects reduced the spacing effect as predicted. The opposite relationship is seen in the right-hand panel. The subjects in the "depressed" group showed a spacing effect for affectively loaded words, but the subjects in the "neutral" group did not. Thus, the effects of spacing depended on both connotation and mood, and this higher order interaction was significant $[\mathrm{F}(2,68)=3.77, \mathrm{p}=.03, \eta=.31]$. An alternative way of describing the interaction shown in Figure 1 is to note that recall under massed practice is elevated and recall under spaced practice is lowered when mood and connotation do not match.

In general terms, the idea that the spacing effect is related to a congruence between the subject's mood and the affect generated by the conditions of practice receives some support. One consequence of such a finding is that the affect generated by different practice conditions enters into the recall process by means of the "mood nodes" that are associated with the to-beremembered items (Bower, 1981; Isen, Shalker, Clark, \& Karp, 1978). This conclusion needs to be tempered by noting that the mood-congruence effect that we obtained depended on word connotation. The nature of this dependency was unexpected. Since a negative mood was induced, Bower's (1981) results lead to the expectation that the kind of results shown in the right-hand panel of Figure 1 should occur only for bad words. Because the results in the right-hand panel represent the recall of both good and bad words, we seem to have obtained a more general mood-congruence effect than the kind reported by Bower. What we have obtained appears to be a mood-congruence effect based on a matching of general affective arousal and not on a match between a particular mood and a particular type of material.

Recently, Clark, Teasdale, Broadbent, and Martin (1983) reported that induced depression and induced elation both lead to differential retention of affectively loaded and neutral words. Under either mood, both good and bad words were recalled better than were neutral words. In addition, their subjects tended to show a mood incongruency effect such that bad words were recalled best by the happy subjects and good words were recalled best by the depressed subjects. This mood incongruency was not seen in the present study, but there are substantial procedural differences between their work and ours that preclude direct comparison. In any event, a general congruence based on affective arousal, connotation, and the conditions of practice has important implications for the way in which theories attempt to account for the representation of affect in memory and how affect operates to influence retention.

\section{REFERENCES}

Beck, A. T. (1967). Depression: Clinical, experimental and theoretical aspects. New York: Harper \& Row.

Bower, G. H. (1981). Emotional mood and memory. American Psychologist, 36, 129-148.

Clark, D. M., Teasdale, J. D., Broadbent, D. E., \& Martin, M. (1983). Effect of mood on lexical decisions. Bulletin of the Psychonomic Society, 21, 175-178.

Elmes, D. G., Dye, C. J., \& Herdelin, N. J. (1983). What is the role of affect in the spacing effect? Memory \& Cognition, 11, 144-151.

Glenbera, A. M. (1979). Component-levels theory of the effects of spacing of repetitions on recall and recognition. Memory \& Cognition, 7, 95-112.

Isen, A. M., Shalker, T. E., Clark, M., \& Karp, L. (1978). Affect, accessibility of material in memory, and behavior: A cognitive loop? Journal of Personality and Social Psychology, 36, 1-12.

Jenkins, J. J. (1960). Degree of polarization and scores on the principal factors for concepts in the semantic atlas study. American Journal of Psychology, 73, 274-279.

Leight, K. A., \& Ellis, H. C. (1981). Emotional mood states, strategies, and state-dependency in memory. Journal of Verbal Learning and Verbal Behavior, 20, 251-266.

Velten, E. A. (1968). A laboratory task for the induction of mood states. Behavior Research and Therapy, 6, 473-482.

Zajonc, R. B. (1980). Feeling and thinking: Preferences need no inferences. American Psychologist, 35, 151-175.

(Manuscript received for publication January 29, 1984.) 